\title{
WINDOW-BASED STATISTICAL ANALYSIS OF TIMING SUBCOMPONENTS FOR EFFICIENT DETECTION OF MALWARE IN LIFE-CRITICAL SYSTEMS
}

\author{
Nadir Carreon \\ Allison Gilbreath \\ Roman Lysecky \\ Department of Electrical and Computer Engineering \\ University of Arizona \\ Tucson, AZ USA \\ \{nadir, alligilbreath, rlysecky\}@ece.arizona.edu
}

\begin{abstract}
Securing life-critical embedded systems, particularly medical devices, requires both proactive security measures that prevent intrusions and reactive measures that detect intrusions. This paper presents a novel model for specifying the normal timing for operations in software applications using cumulative distribution functions of timing subcomponent within sliding execution windows. We present a probabilistic formulation for estimating the presence of malware for individual operations by monitoring the internal timing of the different components of the system, and we define thresholds to minimize false positives based on training data. Experimental results with a smart connected pacemaker and three sophisticated mimicry malware scenarios demonstrate improved performance and accuracy compared to state-of-the-art timing-based malware detection
\end{abstract}

Keywords: Timing-based threat detection, Anomaly detection, Embedded system security, Non-intrusive hardware

\section{INTRODUCTION}

The security of life-critical embedded systems, particularly medical devices, is a critical concern among stakeholders. Having pervasive network access, connected embedded systems (e.g., medical Internet of Things (IoT)) face numerous and wide-ranging security and privacy threats. While IoT devices have grown explosively and are estimated to reach several billions by 2020 (Evans 2013) malware targeting these devices has seen similar growth (McAfee Labs 2016). While regulatory guidance may manage the exposure to security risks and vulnerabilities during design and deployment, it is insufficient to fully secure lifecritical systems throughout their lifecycles. Additionally, while proactive methods (e.g., secure communication protocols) to secure systems during the design stage are essential, the likelihood of zeroday threats cannot be ignored. There are many demonstrations of exploiting vulnerabilities in lifethreatening hacks, including attacking pacemakers, implantable cardiac defibrillators, and insulin pumps. For example, it was demonstrated that by using publicly available information, a malicious packet could be transmitted to an insulin pump that would deliver a fatal dose of insulin (Li, Raghunathan, and Jha 2011).

Once a system is deployed, malware detection is needed to detect when attackers are able to circumvent a system's defenses. Malware detection can be broadly categorized into signature-based detection and anomaly-based detection. Signature-based detection detects malware by matching execution behaviors to a library of known malware, but embedded systems lack the resources needed to utilize these approaches. Anomaly-based detection commonly focuses on monitoring the internal sequence of operations within the system (Holm 2014), where any deviation from the expected sequence would be considered anomalous. However, existing sequence-based anomaly detection does not protect against mimicry malware (Wagner 
and Soto 2002)(Kruegel et al. 2005), which can hide their presence from sequence-based anomaly detection by mimicking the correct execution sequence of the target application/device.

Timing-based anomaly detection improves the detection rate and resilience to mimicry malware by adding timing information to the system model. Time is a critical component in embedded systems and strict timing constraints are often required to ensure system correctness. By monitoring both the internal timing of operations and the execution sequence, timing-based anomaly detection can detect mimicry attacks by observing the resulting changes in system timing. Several approaches (Zimmer et al. 2010)(Yoon et al. 2013)(Lu, Lysecky, and Rozenblit 2017) use the timing of individual operations to detect malware, but these approaches suffer from high false positive rates, a well-known problem in existing anomaly detectors.

In this paper, we present an anomaly-based malware detection method for life-critical embedded systems that uses a window-based statistical model of the normal system behavior using timing subcomponents of monitored operation. Instead of independently focusing on the aggregated timing of operations, the proposed timing model enables the monitoring of each timing subcomponent for each operation within the software application. For each operation, a probabilistic formulation based on cumulative distribution functions (CDFs), is used to estimate the presence of malware for individual timing subcomponents. The malware detection calculates an estimated probability of malware by measuring the percentage of the CDF that falls outside the normal boundaries for each operation and each timing subcomponent. Experiments with a smart connected pacemaker prototype and three mimicry malware scenarios were conducted to evaluate the detection rate, false positive rate, detection latency, area, and power consumption of the presented approach. We further compare our method to a state-of-the-art timing-based malware detection.

\section{RELATED WORK}

Several efforts have been made to develop timing-based anomaly detection. Most of these approaches detect the timing of single operations without considering the execution timing history. These approaches focus on detection accuracy and detection rates, which are both critical aspects of malware detection, and many achieve excellent results. However, many of these approaches incur high false positive rates, and none focus on minimizing the false positive rate or monitoring the timing of the internal subcomponents. In contrast, our presented approach attempts to minimize the false positive rate, maintain a high detection rate, consider the subcomponent timing, and provide a quantitative estimate of the probability of malware.

Patel and Parameswaran (2012), and Patel, Parameswaran and Ragel (2013) presented a MPSoC intrusion detection method that monitors the execution's sequence and time of basic blocks within the software application using a control flow map. The detection method inserts custom instructions in the software binary to identify basic blocks. Those instructions transmit the execution data to a dedicated processor for analysis. The overhead of the instrumented code and additional processors incur a performance overhead between $6.6 \%$ and $44 \%$ and an area overhead of $26.9 \%$. For embedded systems, such as implantable medical devices, the impact on the performance of the application is often infeasible or prohibitively expensive.

Zimmer et al. (2010) proposed a timing-based intrusion detection system at the granularity of function calls and return paths, specifically designed to detect code injection attacks. However, this approach makes decisions based on individual operations and using lumped time measurements. This can lead to a lower detection rate, due to overlapping of the execution time between malware and the normal timing model.

Lu and Lysecky (2017), and Lu, Lysecky, and Rozenblit (2017) developed a range-based runtime anomaly detection method that monitors the best-case execution time (BCET) and worst case execution time (WCET) of individual operations within a software application using both the lumped timing model, and the subcomponent timing model. Although their approach proved the advantages of using the subcomponent timing, they make decisions based on individual operation without considering statistical characteristics of execution timing. By only considering the timing range defined the BCET and WCET of individual events, the timing variability therein presents challenges in accurately training the model to achieve low false positives. 
Yoon et al. (2013) presented SecureCore, which monitors the timing distribution of basic blocks. At runtime, SecureCore measures the execution time of a basic block and estimates the probability of that time from a statically determined timing distribution. If that probability is less than a specified threshold (e.g., $5 \%$ ), SecureCore flags the execution as potentially malicious. This approach is highly dependent on the threshold utilized, since false alarms triggered at runtime may decrease the overall system performance. As such, a balanced threshold is critical for the correct functionality of the system. However, it should be noted that false positives are inherent in this approach, and when applied to operations at a coarse granularity (e.g., system/function calls) the false positive rate can be very high.

\section{ASSUMPTIONS AND THREAT MODEL}

The goal of CDF-based anomaly detection is to detect sophisticated mimicry malware with minimum or no false positives given the following assumptions and threat model.

- The target malware is mimicry malware (Wagner and Soto 2002)(Kruegel et al. 2005), which attempts to evade anomaly detection by mimicking normal execution behavior. Mimicry malware interleaves malicious and normal execution and are sophisticated enough to escape detection from simple sequence-based anomaly detection. Sequence-based anomaly detection is also used in our approach, which can detect non-mimicry malware and necessitates an attacker's need to use mimicry malware.

- The attacker either has access to the system's software or can simulate the system execution to determine the system's execution sequence, which is needed to can create mimicry malware. The attacker is able to remotely insert the malware into the system utilizing software that exploits a vulnerability, which may be unknown or known but unpatched at the time of insertion. The anomalybased malware detection presented in this paper focuses on detecting malware that has already been inserted in the system and not on detecting the software or system vulnerabilities that lead to the intrusion, both which are beyond the scope of this paper.

- The target embedded application consists of multiple software tasks (or threads) executing on a single processor core, although we note that the detection method presented herein can be applied to other application scenarios including multicore systems.

- The granularity detection presented is at the level of system and function calls, called operations. The proposed approach can be applied at different granularities following the same design principles.

- The malware detection is implemented in hardware and interfaces to the main processor core using the microprocessor trace port. The trace port interface allow non-intrusive (i.e., zero performance impact) observation of monitored operations and subcomponent timing measurements. While the detailed hardware implementation is beyond the scope of this paper, our previous malware detection hardware requires approximately $3 \%$ power overhead compared to the base system.

To evaluate the CDF-based anomaly detection method, we consider three mimicry malware based on known malware (albeit from different applications) (Sametinger et al. 2015)(Wasicek, Derler and Lee 2014). The Fuzz malware that is commonly used to interfere with the system's pre-defined functionality by fuzzing (i.e., slightly changing) data (Wasicek, Derler and Lee 2014). Fuzz malware can be implemented in various levels, which enables the evaluation of the effectiveness of malware detection for different fuzzification levels. The Information Leakage malware reads the patient's cardiac activity log and transmits the data to a third-party server. These three malware were implemented by interleaving malicious operations with mimicked normal operations that overall follow the normal execution sequences of the software tasks. Our primary threat is malware affecting legitimate executables, specifically mimicry malware, which assume an attacker knows which operations are monitored. As the approach detects deviations in execution sequences and timing, it can also indirectly detect other malware types. 


\section{TIME ANALYSIS MODELS}

\subsection{Lumped Timing Model}

Previous time based malware detection methods utilize lumped time measurements. The resulting lumped timing model utilizes a single value that combines all elements that have an effect on the time of the operation (i.e., cache misses, interrupts). This value represent the complete time a specific operation (i.e., readSensor, openFile) takes to execute. This value is obtained by monitoring the processor's trace port for the start and end addresses of known function calls. When the instruction address from the trace port matches the start address of a monitored operation, a timer is enabled. Once the instruction address from the trace port matches the end address of an operation, the timer is disabled. The value obtained from the timer (measured in clock cycles), can be translated to a time value, which is defined as the lumped time for the specific operation. The advantage of this model is its simple implementation. However, the disadvantage is the inherent longer time measured by the operation, which increases the risk of an overlap between the malware time, and the normal time at runtime. This would allow the malware to execute without being detected by the proposed CDF malware detection.

\subsection{Subcomponent Timing Model}

The timing of specific operations is affected by the underlying system architecture, operating system, and execution environment, which can lead to unpredictable timing behaviors (e.g., cache behaviors). For example, the execution time of a function call is influenced by the instructions generated during compilation, pipeline structure, cache/memory access delays, interrupts, context switches, etc. Therefore, the timing of events can vary widely, such that detecting malware may be difficult. Fortunately, the information available from the processor's trace port can be utilized to analyze the execution behavior to separate the timing into several subcomponents.

We define two classes of timing subcomponents, namely intrinsic timing and incidental timing. Intrinsic timing is the timing of the software execution in the absence of delays or interference from the system architecture, OS, or other tasks. In other words, the intrinsic timing is the ideal software execution time, which is relatively stable. Incidental timing is the timing due to the execution environment in which the software is executed, and incorporates several subcomponents. Within the current approach, incidental timing subcomponents include I\$ and D \$ misses. We chose these subcomponents due to their inherent ability to detect deviations in the temporal and spatial characteristics of instruction and data addresses caused by malware. By isolating each timing subcomponent, the resulting subcomponent model effectively has tighter bounds, which can be used to increase the detection rate of the CDF based anomaly detector. As shown in Figure 1, the execution time of some previously unknown malware overlapped with the lumped timing model of the system. After splitting this lumped model into the subcomponent model it allows us to detect these malware by looking at the intrinsic timing, and dCache timing.
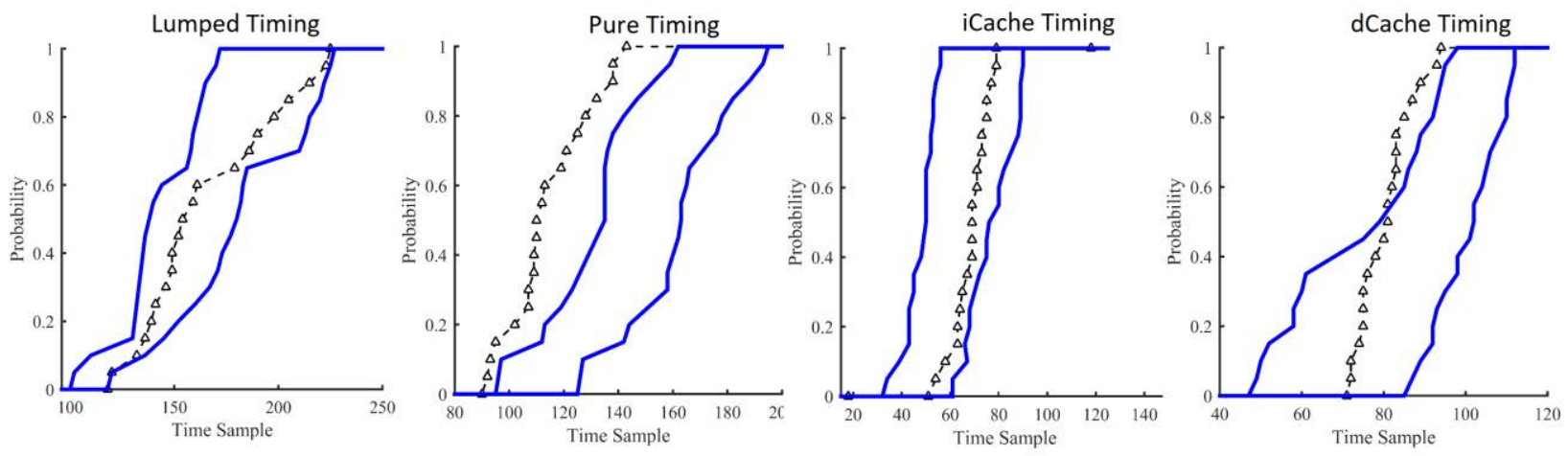

Figure 1: Comparison between the normal timing (blue solid), and the fuzz malware timing for a single execution window using the lumped timing and the subcomponent timing models. 


\section{CDF-BASED ANOMALY DETECTION}

Figure 2 presents the design flow of the CDF-based anomaly detection method. The software application is statically analyzed (Stollon 2011) to determine the operations, $o_{i}$, within all software tasks. For each operation, the system is executed to collect training data by executing the system under normal execution scenarios, for a sufficient duration. For a specific window size and stride, the CDF analysis determines the CDFs per window within the training data. These CDFs are used to calculate the upper and lower bounds Bound $_{\text {upper }}\left(o_{i}\right), \operatorname{Bound}_{\text {lower }}\left(o_{i}\right)$, of the CDF per operation, used to detect deviations at runtime.

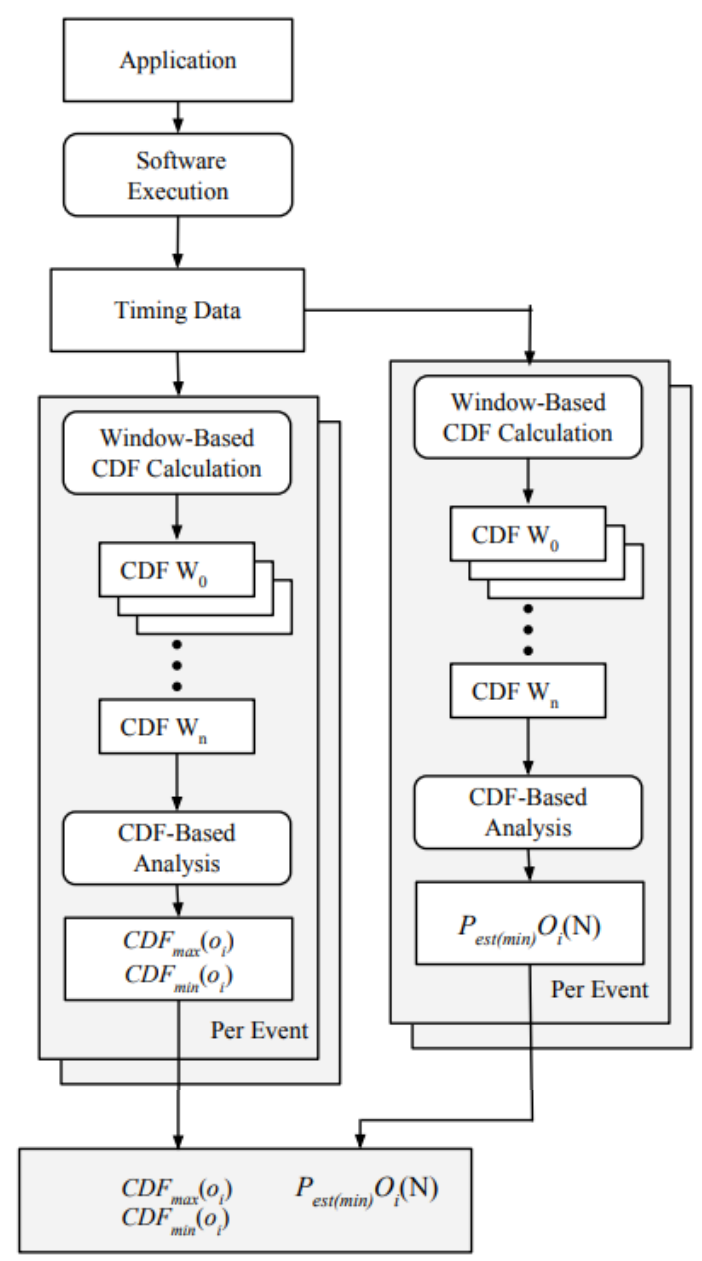

Figure 2: Design flow of the CDF-based anomaly detection. The CDF Analysis module is utilized both at runtime and in static analysis, with the only distinction being the input data will be extracted at runtime, which may or may not be malicious.

This approach is based on the Kolmogorov-Smirnov test (K-S test) (Chakravarti, Laha and Roy 1967) and seeks to detect anomalies by statistically comparing the distribution of timing data between a normal system execution model and the runtime execution. Without assuming a specific distribution, the K-S test can be used to test a sample with a reference distribution for equality, where the reference distribution is the normal system model and the sample is the runtime execution. To test if the sample and normal distribution are equal, the K-S test computes the CDF for both, and measures the maximum difference between the two. While the K-S test can be directly applied to detect anomalous executions, one would need to collect 1000s of timing samples for each operation before testing the distribution, leading to unacceptably long detection latencies. Based on the K-S test, our approach measures the execution timing with a small execution window. To construct the normal system model, the CDF for each execution window in the training data is 
determined. However, instead of storing all CDFs, which would require prohibitively larger memory requirements, only the minimum and maximum boundaries of the CDFs across all windows are stored.

After determining the CDF boundaries, additional normal training data is used to calculate the maximum deviation the CDFs can have from the normal execution while still being considered normal, defined as the threshold. For each operation, the percentage of CDF values for each execution window outside the operation's CDF boundaries is used to determine an estimate of the probability of malware. False positives can be quantified by analyzing the estimated probability of malware for normal execution timing.

At runtime, the detector utilizes the CDF boundaries to estimate the probability of malware for each operation. Timing samples are collected using the same window size and stride. The CDFs for each operation are calculated, and the percentage of CDF values outside the statically determined CDF bounds are used to estimate the probability of malware for each operation. This estimated probability is compared against the predefined threshold to determine if malware is present in the system.

\subsection{Window-based CDF Boundary Construction}

The CDF represents the distribution of timing samples within an execution window, as shown in Figure 3. Creating a model of the normal system behavior using CDFs allows one to estimate the percentage of overlap between runtime CDFs and the normal system model's CDFs. Storing and analyzing the entire execution history for an operation is infeasible and would lead to prohibitively long detection delays. Therefore, the CDF-based anomaly detection collects and analyzes an operation's timing within a fixed execution window that maintains multiple execution timing samples. The window size, defined as the number of timing samples maintained, should be large enough for statistical analysis but small enough to reduce the detection delay. For each window, the stride defines how many new timing samples are collected before re-calculating the CDF. A smaller stride produces smaller changes in the CDF, but requires recalculating the CDFs more frequently to detect malware. However, a larger stride would allow malware to execute longer before being detected, which could be fatal for some systems.

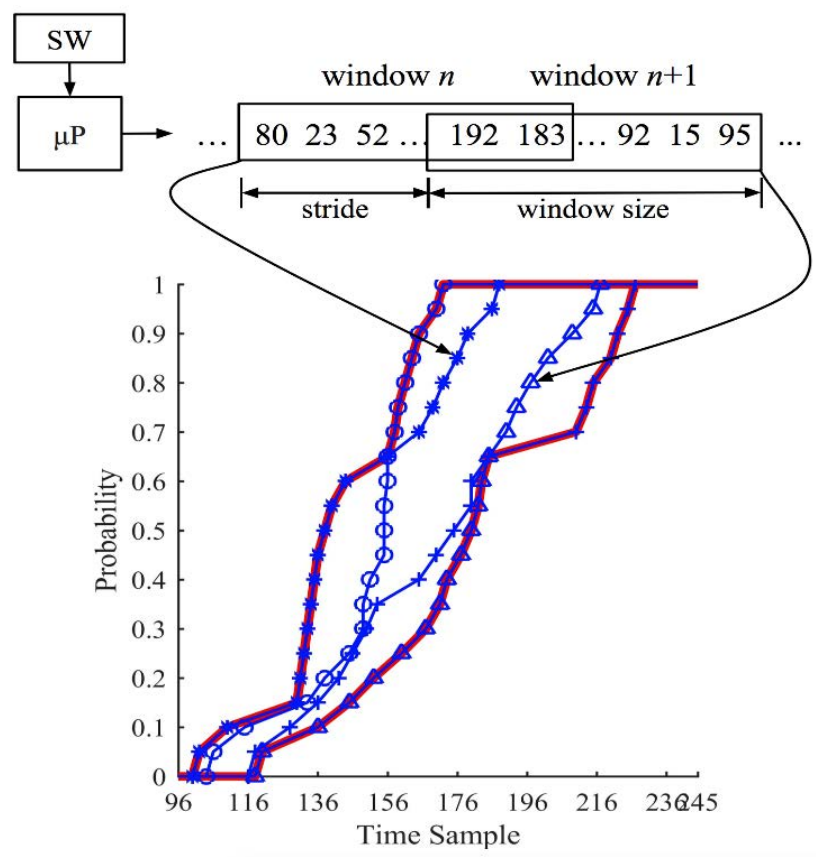

Figure 3: Conceptual overview of window-based CDF calculation, highlighting the sliding windows of timing samples used to calculate the CDF of an operation. The plot shows the CDFs for four different windows, from which the upper and lower CDF boundaries can be calculated.

A conceptual overview of the window-based CDF calculations showing the resulting CDFs for four different execution windows and the resulting boundaries is shown in Figure 3. In this example, the window 
size is 20 and the stride is 5 . Thus, each CDF involves 20 samples, in which $25 \%$ are new timing samples and $75 \%$ are prior samples. After the CDFs for all windows of an operation are obtained during the training stage, the boundaries that define the normal system's model can be determined. The red bolded lines illustrates the CDF bounds. The lower and upper boundaries are constructed by points in the CDFs that have the lowest cumulative probability, and the highest cumulative probability at each timing value respectively. These boundaries are configured into the anomaly detector and used at runtime. Instead of fitting the boundary curve to be a high dimensional representation, because a fixed window size is used, the CDF's cumulative probability will be discretized with a step size equal to the inverse of the window size. Thus, the CDF boundaries are stored as two scalar arrays, Bound $d_{\text {upper }}\left(o_{i}\right)()$ and Bound $d_{\text {lower }}\left(o_{i}\right)()$, that contain the timing values corresponding to each discrete cumulative probability step.

\subsection{Estimating Probability of Malware and Threshold-based Malware Classification}

For an operation $o_{i}$, the estimated probability of malware, $P_{e s t} o_{i}(M)$, depends on the percentage of CDF values outside the CDF boundaries previously defined. Figure 4 presents an example demonstrating how the probability of malware is calculated. The red solid lines are the normal boundaries, and the dashed lines are the CDFs obtained from three different windows of runtime timing data. The black (crosses) CDF is completely outside the CDF boundary, and thus is estimated to have $100 \%$ malicious execution. In contrast, the blue (circles) CDF is completely inside and thus is estimated to have $0 \%$ malicious execution. For a CDF that partially overlaps with the CDF boundary, the probability of malware is estimated as the percentage of points within the $\mathrm{CDF}$ that fall outside the boundaries. For example, the green (triangles) CDF has a probability of malware $P_{e s t} O_{i}(M)=1-(0.65-0.20)=0.55$, which indicates there is estimated to be a $55 \%$ probability the execution is malicious. In practice, with the $\operatorname{Bound}_{\text {upper }}\left(o_{i}\right)()$ and $\operatorname{Bound} d_{\text {lower }}\left(o_{i}\right)()$ arrays, the $P_{\text {est }} o_{i}(M)$ is calculated by determining the number of samples that fall outside these bounds. An operation is considered malicious if the estimated probability of malware, $P_{e s i} O_{i}(M)$ is greater than a predefined threshold.

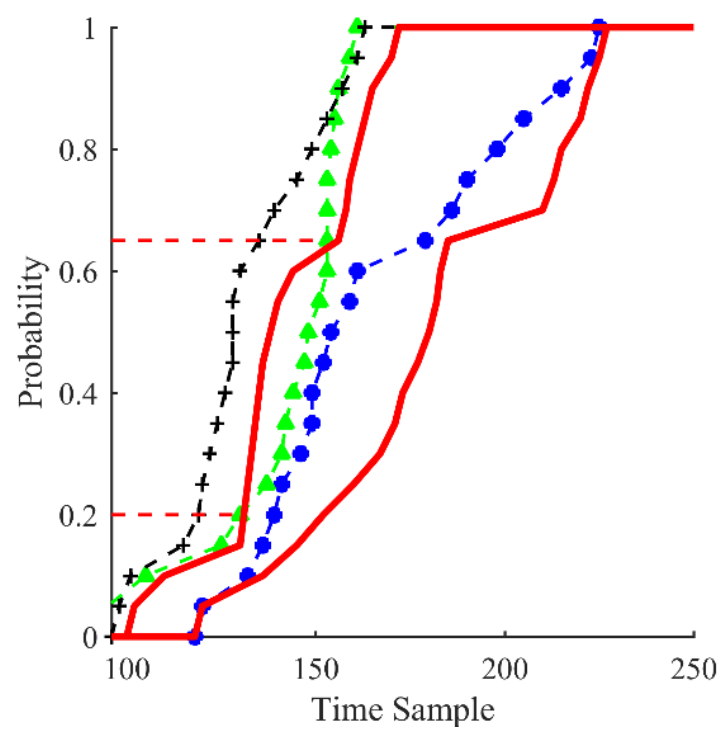

Figure 4: Three runtime CDFs plotted with the upper and lower CDF bounds (red solid lines), showing the overlap used to calculate the estimated probability of malware. Normal execution of the system (blue circles) is always inside the previously obtained boundaries. The CDFs for malware (black crosses and green triangles) results in a CDF that does not fall within the CDF boundaries.

Due to limitations of design-time training, some normal system executions may deviate from the CDF boundaries. Without accounting for these deviations, a high false positive rate would be expected. The threshold is utilized to minimize the false positive rate. For each operation, the threshold is set to the minimum overlap found in the second training data set (normal data only). For example, assume the minimum overlap throughout all windows of operation $o_{i}$ for the second set of normal timing data is 0.90 . 
This means that the highest estimated probability of malware for normal system execution is 0.10 , which in turn means that a runtime estimated probability of malware greater than 0.10 will be reported as malware. This approach strives to ensure the CDF-based anomaly detection is accurate with minimal false positives.

\subsection{Runtime Detection}

At runtime, the threshold of each operation and normal CDF boundaries are configured within the hardware-based malware detector. The malware detector collects timing samples of each operation by analyzing the signals from the processor trace port. Whenever the stride is reached for an operation's window, the detector calculates the $\mathrm{CDF}$ and $P_{e s t} O_{i}(M)$ for the operation, and compares that estimated probability with the threshold $T o_{j}$. If $P_{e s t} o_{j}(M)>T o_{j}$, the detector asserts a non-maskable interrupt indicating the presence of malware.

\section{EXPERIMENTAL RESULTS}

\subsection{Smart Connected Pacemaker}

We developed a smart connected pacemaker and implemented a complete system prototype using the Artix7 XC7A200T FPGA. The pacemaker prototype is representative of a complex embedded system that monitors, analyzes, stores, and transmits data, while providing life and safety critical operations. The pacemaker, shown in Figure 5, includes a simulated heart, a tremor sensor, an impulse pacer, and four timers. The simulated heart beats irregularly and reacts to the impulse pacer controlled by the pacemaker's software. The cardiac activity sensor interfaces to the simulated heart and sends the measured activity to the microprocessor via interrupts. The output from the cardiac activity sensor also controls the AtrioVentricular Interval (AVI) and the Ventricular-Atrial Interval (VAI) timers. These timers are used to maintain the appropriate delay between the atrial/ventricular and ventricular/atrial activation and will generate an interrupt if the AVI/VAI exceeds a specific interval configured by a physician. The PVARP/VRP timers filter noise in the ventricular and atrial channels, respectively (Jiang et al. 2012)(Singh, Wellings and Cavalcanti 2012).

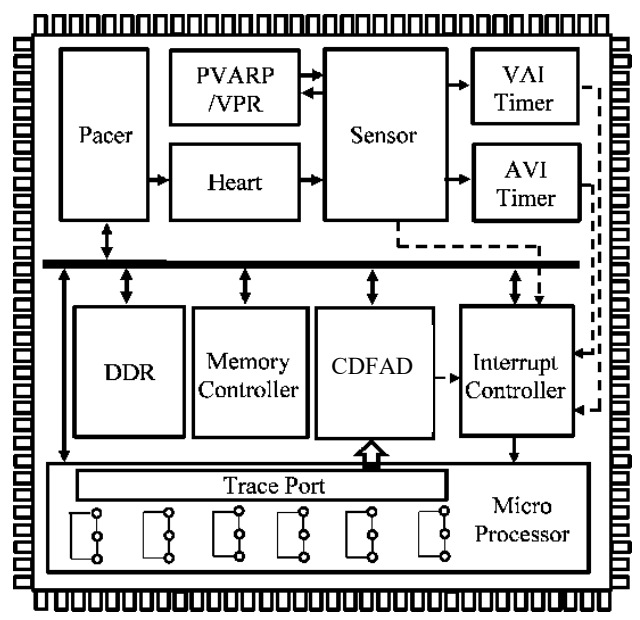

Figure 5: Smart connected pacemaker system architecture.

The pacemaker's software consists of three tasks, named Calculation Task, Analysis Task, and Communication Task, and four interrupt service routines (ISRs). The ISRs interact with the pacemaker's cardiac activity sensor and timers. ISR operations include performing the atrial and ventricular pacing and recording ventricular and atrial activity. The first software task calculates the Upper Rate Interval (URI) and records cardiac activity to a daily log file. A second software task analyzes the cardiac activity and detects a high URI, which indicates the pacemaker cannot pace the heart correctly or that pacemaker's cardiac activity sensor has malfunctioned. In the operation of a high URI, the pacemaker immediate 
transmits a warning message to alert the physician. The software task is responsible for communication, by which the physician can configure the pacemaker, or a monitoring device can access cardiac activity logs.

\subsection{Detection Rate and False Positive Rate}

Using the four mimicry malware, the detection and the false positive rates of the CDF-based anomaly detection are evaluated. The true positive rate (TPR) (i.e., detection rate) is calculated as the number of malware executions classified as malware, divided by the total number of malware executions equation.

$$
T P R=\frac{\text { Malware executions classified as malware }}{\text { Total malware executions }} .
$$

Using a separate set of data, the false positive rate (FPR) is calculated as the number of normal executions classified as malware divided by the total number of normal executions.

$$
F P R=\frac{\text { Normal executions classified as malware }}{\text { Total normal executions }} .
$$

\subsection{Malware Detection Performance}

Figure 6 presents the average results for all monitored operation across all software tasks. For the FPR, both the intrinsic and I\$ subcomponents have FPRs less than 3.88\%, which is lower than the lumped timing model's FPR of $8.19 \%$. In contrast, the average FPR for the D\$ subcomponent is the highest, with an average FPR of $16.98 \%$. To reduce the FPR for this subcomponent, additional training data could be collected to improve the bounds (although such analysis is left as future work). Additionally, the results help to isolate which subcomponents contribute to the overall higher FPR for the lumped timing model.

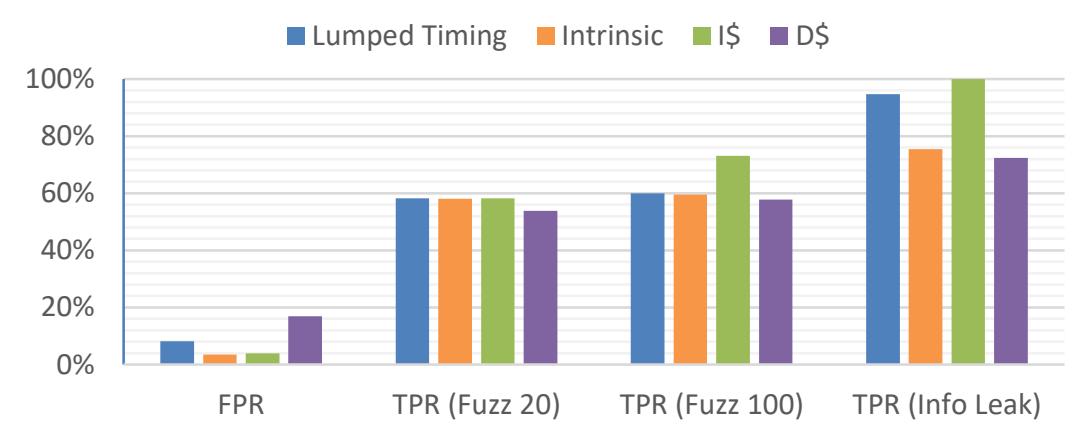

Figure 6: Average FPR and TPR for Fuzz 20\%, Fuzz 100\%, and Information Leakage malware (averaged across all monitored operation and software tasks).

Considering a single subcomponent, the I\$ subcomponent yields the highest TPRs of $58.21 \%, 73.05 \%$, and $100 \%$ for the Fuzz 20, Fuzz 100, and Information Leakage malware, respectively. For a single subcomponent, D\$ alone has the lowest TPR of 53.91\%, 57.78\%, and $72.42 \%$, respectively. Compared to the lumped timing approach, using the I\$ subcomponent yields a $13.05 \%$, and $5.33 \%$ increase in the TPR for the Fuzz 100 and Information Leakage respectively malware, and a 0.01\% reduction in the Fuzz 20.

We further analyzed the FPR and TPR for specific tasks within the smart connected pacemaker, specifically focusing on tasks affected by each malware. Figure 7 presents the average FPR and TPR for the monitored operations within the Calculation Task for the Fuzz 20 and Fuzz 100 malware. The lumped timing models' FPR is $9.58 \%$, while the FPRs for intrinsic and I\$ are 4.82\% and 4.85\%. For the Fuzz 20 malware, the lumped timing model achieves a higher TPR than any individual timing subcomponent $(66.55 \%$ versus $51.68 \%$ ), but for the Fuzz 100 malware the I\$ subcomponent yields the highest TPR (68.2\% versus 64.4\%). 


\section{Carreon, Gilbreath and Lysecky}

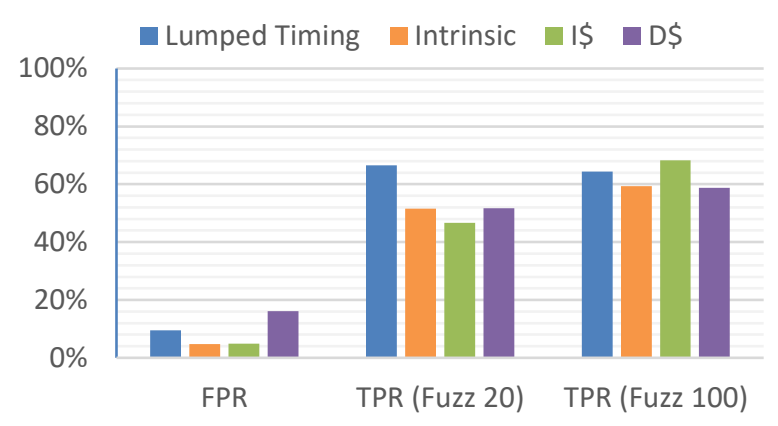

Figure 7: Average FPR and TPR for the Calculation Task for the Fuzz 20 and Fuzz 100 malware.

Figure 8 presents the average FPR and TPR for the monitored operations in the Information Task considering the Information Leakage malware. Across all timing models, the FPR remains low, with FPRs of $1.59 \%, 0 \%, 3.17 \%$, and $4.76 \%$ for the lumped timing, intrinsic, I timing, and D \$ timing, respectively. The lumped and intrinsic subcomponents have the lowest FPR. The I\$ subcomponent again has the highest TPR of $100 \%$, which is a $5 \%$ increase over the lumped timing model.

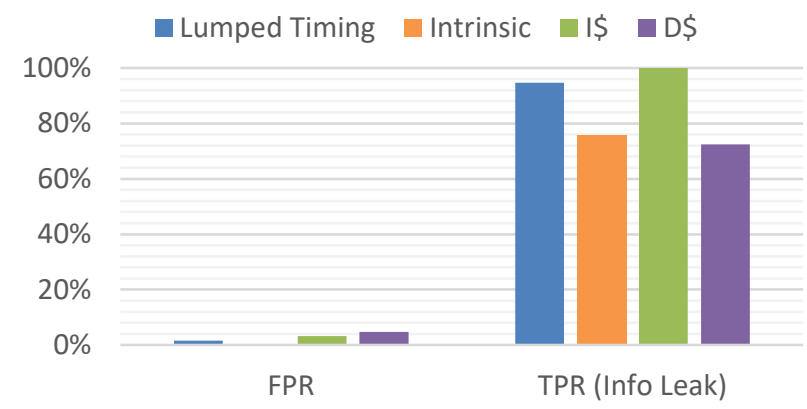

Figure 8: Average FPR and TPR for the Information Task for the Information Leakage malware.

Finally, Figure 9 presents the average FPR and TPR for monitored operations in the Communication Task considering the Fuzz 20 and Fuzz 100 malware. The intrinsic and I\$ subcomponents have the lowest FPR and highest TPR. Compared the lumped timing model, the I\$ subcomponent achieves a $6 \%$ lower FPR and a 15\% higher TPR for the Fuzz 20 malware, and yields a 20\% increase in TPR for the Fuzz 100 malware.

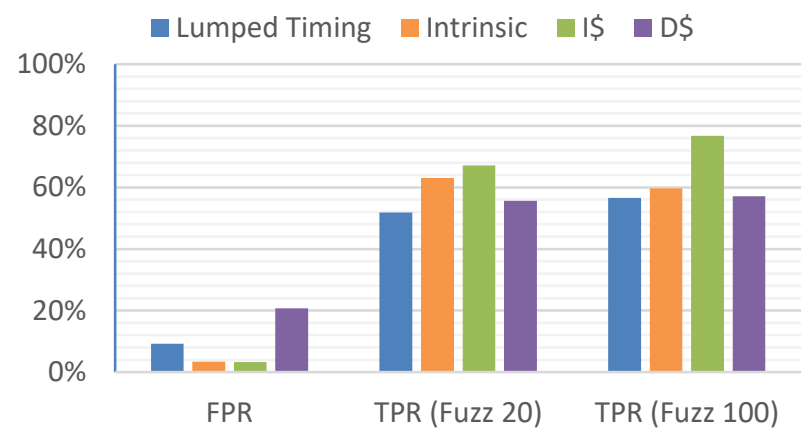

Figure 9: Average FPR and TPR results for the Communication Task for the Fuzz 20 and Fuzz 100 malware. 
Instead of utilizing a single operation for the malware classification, the classification results for each operation can be combined, such that the system execution is considered malicious if at least one operation in the sequence of operations for a software task (or across all software tasks) is malicious. However, without careful selection of the monitored events, this approach could result in a higher FPR. Thus, to evaluate this approach, we only consider operations whose individual FPR is less than 5\%. Using this approach, the lumped timing, intrinsic timing, I\$ timing, and D\$ timing yield TPRs of 99.8\%, 92.0\%, $99.29 \%$, and $99.90 \%$, respectively, and FPRs of $6.32 \%, 1.83 \%, 11.88 \%$ and $6.88 \%$, respectively. In this approach, the intrinsic timing subcomponent yields the lowest FPR (1.83\%), at the expense of a reduction in the detection rate of $\sim 7.07 \%$ in average compared to the subcomponents and lumped.

\section{CONCLUSIONS AND FUTURE WORK}

We presented an anomaly-based malware detection method that combines windows-based statistical analysis with a subcomponent timing model. For malware classification at the operation level, the I\$ timing subcomponent yields the highest TPR and second lowest FP, with an average decrease in FPR of $4.31 \%$ and an increase in TPR of $6.12 \%$ compared to the previous lumped timing model. For task or level system malware classification, the intrinsic timing subcomponent yields the best tradeoff of FPR and TPR, achieving an FPR of $1.83 \%$ and TPR of $92.0 \%$. Future work includes investigating the impact of additional training data, which can be collected during software certification efforts, to reduce the false positive rate for the other subcomponent. Future work also includes evaluating the advantage of path-based malware classification that defines thresholds based on the execution of all monitored operations within each execution path, rather than independently for each operation.

\section{ACKNOWLEDGEMENTS}

This research was partially supported by the National Science Foundation under Grant CNS-1615890.

\section{REFERENCES}

Arora D, A. Raghunathan, S. Ravi, N. Jha. Architectural Support for Safe Software Execution on Embedded Processors. Conf on Hardware Software Co-design and System Synthesis, pp.106-111, 2006.

Carreon N., Lu S., Lysecky R. Hardware-based Probabilistic Threat Detection and Estimation for Embedded Systems. IEEE International Conference on Computer Design (ICCD), 2018.

Chakravarti, Laha, and Roy, (1967). Handbook of Methods of Applied Statistics, Volume I, John Wiley and Sons, pp. 392-394.

Evans, D. The Internet of Things: How the Next Evolution of the Internet Is Changing Everything. Cisco White Paper, 2013.

Holm H. Signature Based Intrusion Detection for Zero-Day Attacks: (Not) A Closed Chapter? Hawaii International Conf. on System Sciences, 2014.

Jiang, Z., M. Pajic, S. Moarref, R. Alur, R. Mangharam. Modeling and Verification of a Dual Chamber Implantable Pacemaker. Conf. on Tools and Algorithms for the Construction and Analysis of Systems, pp. 188-203, 2012.

Kruegel, C. E. Kirda, D. Mutz, W. Robertson, G. Vigna. Automating Mimicry Attacks using Static Binary Analysis. USENIX Security Symposium, pp.161-176, 2005.

Li, C., A. Raghunathan, N. K. Jha. Hijacking an insulin pump: Security attacks and defenses for a diabetes therapy system. Conference on e-Health Networking Applications and Services, pp. 150-156, 2011.

Lu, S., Lysecky R., Rozenblit J. Subcomponent Timing-Based Detection of Malware in Embedded Systems. IEEE International Conference on Computer Design (ICCD), pp. 17-24, 2017. 
Lu, S., R. Lysecky. Time and Sequence Integrated Runtime Anomaly Detection for Embedded Systems. ACM Transactions on Embedded Computing Systems, 17(2), Article 38, pp. 1-27, 2017.

McAfee Labs. Threats Report: December, 2016.

Patel K., S. Parameswaran, SHIELD: A Software Hardware Design Methodology for Security and Reliability of MPSOCs. Design Automation Conference, pp. 858-861, 2008.

Patel, K., S. Parameswaran, R. Ragel. Architectural Frameworks for Security and Reliability of MPSoCs. IEEE Transactions on Very Large Scale Integration Systems, No. 99, pp. 1-14, 2010.

Sametinger J., J. Rozenblit, R. Lysecky, P. Ott. Security Challenges for Medical Devices. Communication of ACM, 58(4), pp.74-82, 2015.

Singh, N.K., A. Wellings, A. Cavalcanti. The Cardiac Pacemaker Case Study and its Implementation in Safety-Critical Java and Ravenscar Ada. Workshop on Java Technologies for Real-time and Embedded Systems, 2012.

Stollon, N. On-Chip Instrumentation: Design and Debug for Systems on Chip. Springer US, 2011.

Wagner, D., P. Soto. Mimicry Attacks on Host based Intrusion Detection Systems. Conf. on Computer and Communications Security, pp. 255-264, 2002.

Wasicek A., P. Derler, E. A. Lee, Aspect-oriented Modeling of Attacks in Automotive Cyber-Physical Systems, Design Automation Conference, pp. 1-6, 2014.

Wilhelm, R., J. Engblom, A. Ermedahl, N. Holsti, S. Thesing, D. Whalley, G. Bernat, C. Ferdinand, R. Heckmann, T. Mitra, F. Mueller, I. Puaut, P. Puschner, J. Staschulat, P. Stenstrom. The Worst-Case Execution-Time Problem - Overview of Methods and Survey of Tools. ACM Transactions on Embedded Computing Systems, 7(36), pp. 1-47, 2008.

Xilinx, Inc. MicroBlaze Processor Reference Guide, UG984. 2016.

Yoon M.-K., S. Mohan, J. Choi, J.-E. Kim, L. Sha. SecureCore: A Multicore-based Intrusion Detection Architecture for Real-Time Embedded Systems. Real-Time and Embedded Technology and Applications Symposium, 2013.

Zhang, T., X. Zhuang, S. Pande, W. Lee. Anomalous Path Detection with Hardware Support. Conference on Compilers. Architectures and Synthesis for Embedded Systems, pp. 43-54, 2005.

Zimmer, C., B. Bhat, F. Mueller, S. Mohan. Time-Based Intrusion Detection in Cyber-Physical Systems. ACM/IEEE International Conference on Cyber-Physical Systems (ICCPS), pp. 109-118, 2010.

\section{AUTHOR BIOGRAPHIES}

NADIR CARREON is a Ph.D. student in Electrical and Computer Engineering at the University of Arizona. His research interests are in embedded systems with emphasis on security and threat detection in medical devices. He received his bachelor's degree in Mechatronics Engineering from Universidad de Sonora, in Hermosillo, Sonora, Mexico in 2014. Email: nadir@email.arizona.edu.

ALLISON GILBREATH is an undergraduate student at the University of Arizona majoring in Computer Engineering and Computer Science. Her research interests are in cybersecurity and data analytics in medical systems and devices. Email: alligilbreath@email.arizona.edu

ROMAN LYSECKY is a Professor of Electrical and Computer Engineering at the University of Arizona. He received his Ph.D. in Computer Science from the University of California, Riverside in 2005. His research focuses on embedded systems with emphasis on medical device security, automated threat detection and mitigation, runtime adaptable systems, performance and energy optimization, and nonintrusive observation methods. Email: rlysecky@ece.arizona.edu 